\title{
Draussen vor dem Gesetz
}

\section{Marginalien zur Raumsemantik in Kafkas Türhüter-Parabel}

Andreas Mauz

\begin{abstract}
»Man muss eben den Mechanismus kennen<,
\end{abstract} sagte sich Karl.»

Frank Kafka, Der Verschollene (1911-14)

»Hier konnte niemand sonst Einlass erhalten, denn dieser Eingang war nur für Dich bestimmt. Ich gehe jetzt und schliesse ihn. «" Kafkas Parabel Vor dem Gesetz (1914) endet vor dem Gesetz. In einer performativen Geste lässt der Autor das Textende koinzidieren mit der Schliessung der Tür, die in das Gesetz führt. Der "Mann vom Lande" (291), der mit der Bitte um Einlass vor den Türhüter tritt, um nach zermürbenden Gesprächen und scheiternden Bestechungsversuchen schliesslich vor dem Gesetz den Tod zu finden, hat in Kafkas Werk unzählige Verwandte. Der Versuch von A nach B zu gelangen, von einem Äusseren in ein Inneres oder umgekehrt, dabei aber mit schwer oder nicht überwindbaren Hindernissen konfrontiert und damit - in jeder Hinsicht - ausgeschlossen zu sein, kann als strukturelle Konstante seines Schreibens gelten. Prominentestes Beispiel ist neben Vor dem Gesetz zweifellos der Schloss-Roman, der den - scheiternden - Versuch des Eintritts ins Schloss auf einer Länge von 300 Seiten entfaltet. Varianten derselben Grundkonstellation bieten, um nur einige zu nennen, die Prosaminiaturen Eine kaiserliche Botschaft, Die Brücke, Der Aufbruch, Heimkehr, Gibs auf! oder Das nächste Dorf:

"Mein Grossvater pflegte zu sagen: ‘Das Leben ist erstaunlich kurz. Jetzt in Erinnerung drängt es sich mir so zusammen, dass ich zum Beispiel kaum begreife, wie ein junger Mensch sich entschliessen kann, ins nächste Dorf zu reiten, ohne zu fürchten, dass - von unglücklichen Zufällen ganz abgesehen - schon die Zeit des gewöhnlichen, glücklich ablaufenden Lebens für einen solchen Ritt bei weitem nicht hinreicht. «" ${ }^{2}$

Dieser Anlage entsprechend erhalten Schwellenräume, Übergangszonen, Orte, an denen ein Innen und ein Aussen, ein Oben und ein

${ }^{1}$ Franz Kafka, Der Process, in der Fassung der Handschrift, hrsg. von Malcolm Pasley, Frankfurt a/M 1990, 294f. Nachweise von Zitaten aus dem Process erfolgen nach dieser Ausgabe direkt im Text.

${ }^{2}$ Franz Kafka, Erzählungen, hrsg. von Max Brod, Frankfurt a/M 1983, 128. 
Unten, ein Dies- und ein Jenseits sich berühren, eine herausragende Bedeutung. Die genannten Texte sind Schwellendramen, angesiedelt an Türen, Toren, Fenstern, Brücken und Treppen. Eher selten gelingt der Eintritt in problemloser Weise, weil - wie im Romanfragment Der Verschollene - eine wohlgesonnene Hotelangestellte "den Mechanismus kennt", den Helden in eine Vorratskammer geleitet und mit Speck, Brot und Bier versieht. Wahrscheinlicher ist, dass er, der verwandelte Gregor Samsa etwa, im Nebenzimmer isoliert, Zeuge der ihn betreffenden Gespräche der Familie wird und beim Versuch, das Zimmer zu verlassen, im Türrahmen steckenbleibt oder dass die Brücke (wie in Die Brücke) sich ihrer Funktion verweigert und den Reisenden in die Tiefe stürzen lässt. Wenn der Eintritt des Helden denn gelingt, so erwartet ihn im Inneren meist nichts Gutes, im Falle des Process etwa ein unerwartetes Verhör. Und auffällig oft sind es hier Türhüterinnen, die Josef K. an der Schwelle empfangen, ohne Speck, Brot und Bier.

Freilich ist die Bewegung im Raum, der Umstand, dass Figuren über präzis bestimmte und limitierte Bewegungsradien verfügen, ein Moment, das für erzählende Texte insgesamt von erheblicher Bedeutung ist. Dies zeigen - in je eigener Weise - Gaston Bachelards psychoanalytisch und phänomenologisch orientierte Poétique de l'espace (1957) ebenso wie Jurij M. Lotmans raumbezogene Erzählanalyse (in: Die Struktur literarischer Texte, 1970) oder neuere Entwürfe literarischer Kartographien. Lotman geht gar davon aus, dass die Existenz einer klassifikatorischen Grenze dem Text überhaupt erst das Potential narrativer Dynamik verleiht. Ein »Sujet» (dies der Lotmansche Begriff für die globale Struktur einer Handlung) entsteht allererst durch das Vorhandensein einer erzählten Welt, die in (mindestens zwei) komplementäre Räume zerfällt, wobei sich die Grenze zwischen diesen Räumen für eine Figur als durchlässig, für eine andere aber als undurchlässig erweist. Der komplementäre Gegensatz zwischen den Teilräumen kann dann auf drei Ebenen entfaltet werden: einer topologischen, welche die erzählte Welt durch die Oppositionen hoch/tief, innen/aussen differenziert, einer semantischen, die sich wertend an sie anschliesst (gut/böse, vertraut/fremd, natürlich/künstlich etc.) und schliesslich einer topographischen, welche die semantisch aufgeladene topologische Ordnung in der dargestellten Welt konkretisiert (Berg/Tal, Stadt/Land, Himmel/Hölle etc.). Die räumliche Ordnung der erzählten Welt ist nach Lotman nicht 
weniger als das »organisierende[...] Element [...], um das herum sich auch die nichträumlichen Charakteristika ordnen « ${ }^{3}$.

Wie weit der Lotmansche Ansatz im Falle Kafkas trägt, wäre im Detail zu untersuchen. Die Vermutung liegt jedoch nahe, dass seine strukturalistische Statik und Strenge erheblich strapaziert würde. Das Bedürfnis nach säuberlicher Trennung - oben der gute Himmel, unten die böse Hölle - wird von Kafka nur sehr bedingt bedient. Die eigentümliche Kraft seiner Texte verdankt sich vielmehr gerade Denk- und Schreibbewegungen, welche die Verpflichtung zur binären, am Satz vom Widerspruch und vom ausgeschlossenen Dritten orientierten Logik oft hinter sich lassen. Was Walter Benjamin Kafkas "wolkige Stellen« nennt, wird Gerhard Neumann folgend gelegentlich auch auf die Formel des "gleitenden Paradoxes « ${ }^{4}$ gebracht. Selbst das hermeneutische Ärgernis des Paradoxes wird überboten. Kafka bringt es ins Gleiten, indem er die Umkehrung (als Auflösung der fixiert paradoxen Struktur) mit einer Ablenkung verschränkt. Die >Richtigstellung< ist dann "nicht einleuchtender als die >verkehrteく Form des Satzes ${ }^{5}$, und auch die Möglichkeit der Beruhigung in einer dialektischen Synthese entfällt. Das erste der Paralipomena zu der Reihe Er kann diese Struktur auf kleinstem Raum verdeutlichen: »Er hat den Archimedischen Punkt gefunden, hat ihn aber gegen sich ausgenützt; offenbar hat er ihn nur unter dieser Bedingung finden dürfen. ${ }^{6}$ Der archimedische Punkt, per definitionem der Ort, um die Welt aus den Angeln zu haben, hebt hier (Umkehrung) nicht die Welt, sondern das Ich aus den Angeln; die Bedingung des Auffindens des Punktes führt (Ablenkung) die Definition seiner selbst ad absurdum.

Was im Folgenden unter dem Stichwort der Raumsemantik angemerkt wird, hat mit dem Lotmanschen Ansatz daher wenig gemein. Vielmehr sollen dem Parabeltext folgend einige Beobachtungen zu dessen Raum- und Bewegungsarrangements benannt werden, die sich unter punktuellem Einbezug des RomanKontextes $\mathrm{zu}$ einer raumsemantisch konturierten These verdichten

\footnotetext{
${ }^{3}$ Jurij M. Lotman, Die Struktur literarischer Texte, München ${ }^{4} 1993,316$.

4 Vgl. Gerhard Neumann, Umkehrung und Ablenkung: Franz Kafkas »Gleitendes Paradox", in: DVJs, Sonderheft: Literatur des 20. Jahrhunderts, 42 (1968), 702-744. Erneut abgedruckt in: Heinz Politzer (Hg.), Franz Kafka, Darmstadt 1973 (= Wege der Forschung, Bd. CCCXXII), 459-515.

${ }^{5}$ Ebd., 464.

${ }^{6}$ Franz Kafka, >Hochzeitsvorbereitungen auf dem Lander und andere Prosa aus dem Nachlass, hrsg. von Max Brod, Frankfurt a/M 1983, 303.
} 
lassen: Die Parabel wird lesbar als zwiespältige Allegorie des Kommentars.

\section{Vor dem Gesetz}

Der Mann, der um Einlass ins Gesetz bittet, kommt von aussen, "vom Lande«; man kann ihn, wie die judaistisch orientierte KafkaForschung betont, als "Am ha-Arez« verstehen, als einen in der Tora ungebildeten Menschen, der in talmudischer Tradition als »Mann vom Lande« bezeichnet wird. Das assoziationsoffene `Gesetz` wird entsprechend zum Innen. Vor dem Gesetz trifft der Mann vom Lande auf den Türhüter, der selbst gleichfalls nicht im Gesetz ist, es möglicherweise nie war (vgl. 298f), dieses aber repräsentiert. Diesem Repräsentanten unterwirft sich der Mann vom Lande im Laufe des ersten Gesprächs, freiwillig; durch seine - falschen - Vorurteile ist er vorverurteilt. ${ }^{7}$ Die Bewegung kommt vor dem Gesetz zum Stehen, der Mann setzt sich und affirmiert damit endgültig jene Anordnung, die erst in seinem Tod noch einmal modifiziert wird. Das zeitdekkende wird in ein zeitraffendes Erzählen überführt, an die Stelle des Einmaligen tritt die Wiederholung: »Dort sitzt er Tage und Jahre.« (293)

Das Gesetz selbst wird allegorisch verdinglicht als Raum eingeführt, als eine Art Gebäude, das eine bestimmte innere Struktur aufweist: eine konzentrische Stufung verschiedener Säle, die jeweils wiederum durch Türen und Türhüter voneinander separiert und miteinander verbunden sind - so zumindest stellt es sich dar, wenn man den Ausführungen des Türhüters folgt, denn zu einem faktischen Eintritt ins Gesetz kommt es ja nicht. (Selbst wenn man sich an die Aussagen des Türhüters hält, so reichen seine Kenntnisse, siehe unten, nicht über das dritte Tor hinaus.)

Aber gerade der spekulative Einblick legt, orientiert man sich einmal an den Innen-Aussen-Relationen, eine Folgerung nahe, welche die scheinbare Positivität des glückenden Eintritts ins Gesetz erheblich relativiert. Denn wenn das Innere des Gesetzes der Schilderung des Türhüters gemäss strukturiert ist, so erfolgte mit dem Eintritt ein paradoxer Umschlag. Verbirgt sich hinter der Tür, welche der Türhüter hütet, nur eine weitere Tür, die von einem wei-

\footnotetext{
7 Dies eine der Pointen von Derridas Lektüre der Parabel. Vgl. Jacques Derrida, Préjuges. Devant la loi, in: ders. et al., La Faculté de juger, Paris 1985, 87-139 bzw. Préjuges. Vor dem Gesetz, Wien 1992.
} 
teren Türhüter gehütet wird, der jedoch ungleich mächtiger ist als der erste, und hinter dieser Tür wiederum eine weitere etc. (die Formulierung »Schon den Anblick des dritten kann nicht einmal ich mehr ertragen« [293] lässt vermuten, dass beim dritten das Innerste noch lange nicht erreicht ist), dann hätte die Annäherung viel von einer Entfernung an sich, da die Wahrscheinlichkeit eines Eintrittes mit jeder Stufe geringer würde und eine Ende dieser Stufen an sich ungewiss. Jeder Schritt bedeutete dann nicht nur, an eine strukturell identische Stelle zu treten, sondern sich einer Art Zentrifugalbewegung auszusetzen.

Die Aussagen des Türhüters über die Beschaffenheit des Inneren des Gesetzes geben jedoch auch zu einer weiteren raumsemantisch relevanten Spekulation Anlass. Der Türhüter ist nach eigener Aussage "nur der unterste« (293). Diese Angabe erlaubt zwei Auslegungen: eine übertragene - der Türhüter als unterster in der Hierarchie der Türhüter - und eine, welche die Verortung im eigentlichen, in einem räumlichen Sinn versteht. Die zweite führt zur Annahme, dass das Gesetz nicht nur eine horizontale konzentrische Stufung aufweist, sondern mit jeder Stufe zugleich in die Höhe strebt, demnach als ein turmartiger Bau vorzustellen wäre. Ein Eintritt ins Gesetz würde sich dann zugleich mit einen Aufstieg verbinden. Diese Lesart darf nicht vernachlässigt werden. Einmal nicht, weil die Begegnung mit - offen formuliert - >Autorität « stets eine ist mit einem Oben (das leicht erhöhte Pult des Vorgesetzten unterscheidet sich in dieser Hinsicht nicht vom Berg als dem klassischen Ort der Gottesbegegnung), insbesondere aber dann nicht, wenn man davon ausgehen kann, dass der Parabel ein Subtext unterliegt, die MidraschLegende nämlich, die davon erzählt, dass Moses beim Aufstieg zum Sinai - mit Gottes Hilfe - vier Türhüter-Engel überwinden muss, um erst dann die Tora zu empfangen. ${ }^{8}$ Die subtile Differenz zwischen der Selbstverortung des Türhüters und der Fremdverortung durch den Erzähler, der ihn als »erste[n]« (294) bezeichnet, macht auf die Notwendigkeit der unterscheidenden Lektüre eher aufmerksam, als dass sie sie nivelliert. Einmal sensibel für eine mögliche

\footnotetext{
${ }^{8}$ Vgl. die Erzählung aus dem Midrasch Pesikta Rabbati (zu Ex 24,15f) in: Jakob Winter / August Wünsche, Die jüdische Literatur seit Abschluss des Kanons. Geschichte der jüdisch-hellenistischen und talmudischen Literatur, Trier 1894 (Neudruck: Hildesheim 1965), 447-453 bzw. Ulf Abraham, Mose >Vor dem Gesetz‘. Eine unbekannte Vorlage zu Kafkas >Türhüterlegende〈, in: DVJs 57 (1983), 636-650. (In überarbeiteter Fassung unter dem zutreffenderen Titel Mose >Vor dem Gesetzく. Eine unbekannte Quelle für Kafkas Türhüterlegende auch in: Voigts, ’Vor dem Gesetz‘, 89103.)
} 
Vertikalität des Gesetzes gewinnt ein weiteres Detail an Bedeutung: der Umstand, dass der Mann vom Lande sich bücken muss, "um durch das Tor in das Innere zu sehn« (292). Offenbar ist es eine niedrige Pforte, die in das Gesetz führt. Wenn das Gesetz im Inneren turmartig aufsteigt, so korrespondiert dies mit der Gegenbewegung des Bückens, der Demutsgeste des Eintretenden. (Die Bückbewegung kehrt invertiert am Ende der Parabel wieder, wenn sich der Türhüter niederbeugt, um die letzte Frage des sterbenden Mannes vom Lande anzuhören.)

Die Position des Mannes vom Lande vor dem Gesetz ist jedoch grundsätzlich keine beliebige, sondern sehr präzise bestimmt: »Der Türhüter gibt ihm einen Schemel und lässt ihn seitwärts vor der Tür sich niedersetzen.«(293; meine Hervorhebung, A. M.) Dieses »seitwärts « ist in verschiedener Hinsicht von Interesse. Zunächst ist diese Positionierung keine freie Entscheidung des Mannes, sondern eine, die sich mit dem Willen des Türhüters verbindet, sei es - beide Lesarten sind möglich - im Sinne einer klaren Platzanweisung oder im Sinne einer Duldung an der Seite, nicht aber in frontaler Ausrichtung. Zweitens verdient das »seitwärts« aber auch Aufmerksamkeit, weil es - ein Detail, möchte man meinen - in den Ausführungen des schrifttreuen Geistlichen hartnäckig Erwähnung findet (vgl. 297, 300; Näheres zum Auslegungsgespräch folgt unten). Soweit die Beobachtungen am Text. Dass diese eigentümliche Insistenz eingehenderer Untersuchung Wert sein könnte, mag eine Passage aus Kafkas Tagebucheintrag vom 4. Dezember 1913 andeuten. Sie skizziert eine Situation, die derjenigen vor dem Gesetz in erstaunlicher Weise gleicht und daher für die Frage nach der Bewertung der Platzwahl/Platzanweisung ebenso von Interesse ist wie für die des Schicksals des Mannes insgesamt:

»Die Furcht vor Narrheit. Narrheit in jedem geradeaus strebenden, alles andere vergessen machenden Gefühl sehn. Was ist dann die Nicht-Narrheit? NichtNarrheit ist, vor der Schwelle, zur Seite des Einganges bettlerhaft stehen, verwesen und umstürzen." 9

Zumindest hier wird das Geradeaus-Streben, die frontale Orientierung, zugunsten einer bettlerhaft-demütigen Stehens vor dem »Eingang « verworfen; von der zitierten Passage ausgehend ist die Leitfrage des Auslegungsgesprächs - Wer ist der Getäuschte: der Mann

\footnotetext{
${ }^{9}$ Franz Kafka, Tagebücher 1910-1923, hrsg. von Max Brod, Frankfurt a/M 1983, 247.
} 
vom Lande oder der Türhüter? - klar zu beantworten: Der Mann vom Lande ist es nicht, er ist kein "Narr».

Oder vielleicht doch. Denn anders als in der Tagebuch-Notiz verschiebt sich die Aufmerksamkeit des Mannes vom Lande im Laufe der Jahre immer stärker vom Gesetz weg, um dessentwillen er eigentlich gekommen ist, hin zum Türhüter: Ihm, und nicht dem Gesetz, gilt nun sein Studium. (Eine Bewegung, die sich als realisierte Metonymie bezeichnen liesse: Die Ursache-Wirkung-Substitution hört auf, Substitution zu sein, die Wirkung tritt an die Stelle der Ursache.) Die (jüdische) Tugend des Studiums wird ins Absurde verzerrt:

"Er vergisst die andern Türhüter und dieser erste scheint ihm das einzige Hindernis für den Eintritt in das Gesetz. Er verflucht den unglücklichen Zufall, in den ersten Jahren laut, später als er alt wird brummt er nur noch vor sich hin. Er wird kindisch und da er in dem jahrelangen Studium des Türhüters auch die Flöhe in seinem Pelzkragen erkannt hat, bittet er auch die Flöhe ihm zu helfen und den Türhüter umzustimmen.« (294)

Oder vielleicht doch nicht. Denn erst in diesem Niedergang, da die Kraft seiner Augen nachlässt und er nicht weiss, »ob es um ihn wirklich dunkler wird oder ob ihn nur seine Augen täuschen«, »erkennt er [...] im Dunkel einen Glanz, der unverlöschlich aus der Türe des Gesetzes bricht« (294). Der Niedergang, das KindischWerden, als Voraussetzung der Erleuchtung? - Man hat wiederholt darauf hingewiesen, dass der Mann vom Lande zunächst steht, dann sitzt und schliesslich - im Sterben - liegt, was der Text durch die lakonische Bemerkung, dass »die Grössenunterschiede [...] sich sehr zuungunsten des Mannes verändert« (294) hätten, kommentiert. "Vor seinem Tode« (294) befindet sich der Mann vom Lande natürlicherweise in der Position «vor dem Gesetz«- kaum zufällig sind die beiden Sätze parallel formuliert -, die er zu Beginn durch bewusstes Bücken einnimmt. Ist er damit dem Gesetz näher? Oder radikaler gefragt: Endet die Parabel tatsächlich vor dem Gesetz? - Nicht unbedingt, denn »jetzt« (294) - und dieses Jetzt nimmt das eingangs fallende Jetzt des nicht-möglichen Eintritts auf (vgl. 292) - erkennt er eben den »Glanz, der unverlöschlich aus der Türe des Gesetzes bricht«. Jetzt, da er bis zum Tode geduldig vor dem Gesetz ausgeharrt und auf den Versuch, gewaltsam einzudringen, verzichtet hat, kommt das Gesetz auf ihn zu. Die letzten Worte des Türhüters sind nicht so eindeutig, wie man zunächst meinen mag. »Hier konnte 
niemand sonst Einlass erhalten, denn dieser Eingang war nur für Dich bestimmt. Ich gehe jetzt und schliesse ihn.«(294f) Das kann auch heissen: "Weil Du im Tode in den Eingang eingetreten bist, der nur für Dich bestimmt war, schliesse ich ihn. «" Einlass ins Gesetz zu finden, hiesse dann nicht ins Gesetz eintreten, sondern eine Einlassung bis zum Tod. Das vermeintliche Aussen erwiese sich damit als Innen, während das vermeintliche Innen den Eintretenden wie angedeutet nur zentrifugal in ein äusseres Aussen katapultierte.

Gewissheit ist aber auch hier nicht zu haben. Möglicherweise geht der Glanz nicht aus dem Gesetz hervor, sondern, als blosser Schein, aus der Phantasie des ge- und enttäuscht Sterbenden. Für den Türhüter, der in berufsbedingter Statik "wohl mit dem Rücken zum Eingang« (301) steht, ist er in jedem Fall unsichtbar.

\section{Vor/nach Vor dem Gesetz}

Wenn sich eine Orientierung an der Raumsemantik in der Parabel als aufschlussreich erweist, so kann sie ausserhalb, vor und nach ihr, in gleicher, aber auch in noch einmal anderer Weise angesetzt werden, nämlich als Semantik des Textraums. Der Fokus auf die Beschaffenheit des Raumes in der Textwelt ist zu erweitern auf den Raum des Textes selbst, der gleichfalls ein Innen und ein Aussen, ein Oben und ein Unten kennt. Nicht nur im Fall sogenannt >Konkreter Poesie` (man denke an Mallarmés Un Coup de Dés oder Gomringers "schweigen«) wird die Form des Textes semantisiert. Auch erzählende Texte können das (Un-)Sinnpotential des Schriftraums nutzen, indem etwa die gewohnheitsmässige Linearität und Sukzessivität der Lektüre durch die Integration vertikaler, transversaler oder zirkulärer Textstrukturen (konkret: Mehrspaltigkeit, Akrosticha etc., aber auch Binnenzitate verschiedenster Art) aus der Bahn gebracht und der Rezipient zu einer Simultaneität des Blicks genötigt wird. Proust vergleicht sein Werk bekanntlich einmal mit einer Kathedrale.

Die Türhüter-Parabel, von Kafka dreimal separat publiziert, hat, als Innen, ihr Aussen im unvollendeten Process-Roman. Dass zwischen beiden komplexe Korrespondenzverhältnisse bestehen, ist nur $\mathrm{zu}$ offensichtlich, und entsprechend gehört es zu den klassischen Thesen der Forschung, die Parabel im Sinne einer "mise en abyme«

\footnotetext{
${ }^{10}$ So Bernd Wittes Paraphrase des Satzes der Türhüters. Vgl. Bernd Witte, Das Gericht, das Gesetz, die Schrift. Über die Grenzen der Hermeneutik am Beispiel von Kafkas Türhüter-Legende, in: Klaus-Michael Bodgal, Neue Literaturtheorien in der Praxis. Textanalysen von Kafkas >Vor dem Gesetz‘, Opladen 1993, 94-113, 97.
} 
(Jacques Derrida), eines »inneren Fahrplans« (Heinz Politzer) oder einer "Spiegelerzählung" (Gerhard Kurz) auf den Roman zu beziehen bzw. diesen als »entfaltete Parabel«(Walter Benjamin) zu lesen. Dieser Aspekt kann und braucht hier nicht eingehender besprochen werden, festzuhalten ist lediglich, dass die Rede vom »inneren Fahrplan« etc. nicht in einem strengen Sinn zu verstehen ist. Ebenso deutlich wie die Übereinstimmungen sind die Differenzen zwischen Parabel und Roman, am deutlichsten zweifellos in den verschiedenen Todesarten der Protagonisten. Die Parabel weist hier einen entscheidenden positiven Überschuss auf: Lässt sich der Tod des Mannes auch als Erfüllung auslegen, ist eine derartige Deutung im Falle K.s kaum haltbar. - Im Folgenden nun aber einige Anmerkungen zur unmittelbaren Textnachbarschaft der Parabel, zum Kapitel $» \operatorname{Im}$ Dom«.

Wenn sich am Ende der Parabel die Türe schliesst, wenn der Mann vom Lande ähnlich hilflos vor dem Gesetz liegt, wie der Leser vor dem Vor dem Gesetz- bzw. Process-Text sitzt, dann trifft der Leser auf seinen Repräsentanten im Text, auf Josef K. Wie sich der in den Prozess verstrickte Josef selbst in der Gestalt des Mannes vom Lande begegnet - er fühlt sich "von der Geschichte sehr stark angezogen" (295) -, so begegnet sich der Leser in der Gestalt K.s. Dem dann folgenden Gespräch K.s mit dem Geistlichen (er ist es, der die Parabel erzählt) geht ein Rundgang K.s durch den Dom voran, den er im Auftrag seines Vorgesetzten einem italienischen Geschäftsfreund zu zeigen hat bzw. hätte, denn der Italiener bleibt aus, und Josef hat Zeit, sich im Innern des Doms umzusehen. Der sakrale Raum erscheint vollständig entzaubert. Ihn umgibt eine auffällige Leere (vgl. 279), und nahezu leer ist auch sein Inneres; K. vergisst zunächst, sich zu bekreuzigen; der Bau ist nichts weiter als eine »städtische[...] Sehenswürdigkeit[...]«(288), die man mit einem »Album«, nicht aber mit einem "Gebetbuch" (288) in der Hand besucht und deren Altarbilder nur mittels einer mitgebrachten Taschenlampe betrachtet werden können. - Unerwarteterweise scheint dann aber dennoch ein gottesdienstlicher Vollzug in Gang zu kommen:

„Sollte jetzt etwa eine Predigt stattfinden? In der leeren Kirche? [...] Konnte K. allein die Gemeinde darstellen? Wie, wenn er ein Fremder gewesen wäre, der nur die Kirche besichtigen wollte? Im Grunde war er auch nichts anderes. Es war unsinnig daran zu denken, dass gepredigt werden sollte, jetzt um elf Uhr, an einem Werktag [...].«(284f) 
"Josef K.!« (286) - Die plötzliche individuelle Anrede des Geistlichen (korrespondierend mit der Individualität des Eingangs ins Gesetz) lässt am Adressaten der `Predigt< jedoch keinen Zweifel. Nach längerem Erwägen einer Flucht stellt sich K. der Rede des Geistlichen, der sich als Angehöriger des Gerichts erweist. Er nimmt dessen Informationen über den - schlechten - Stand und die paradox gleitende Verlaufsstruktur des Prozesses - »Das Urteil kommt nicht mit einemmal, das Verfahren geht allmählich ins Urteil über" (289) entgegen. Erst dann verlässt der Geistliche auf K.s Wunsch die Kanzel und zitiert, um Josef über seine das Gericht betreffende "Täuschung" (292) aufzuklären, die "Geschichte» (300), die den »einleitenden Schriften zum Gesetz " (292) entstammt. Vor dem Gesetz steht also vor dem Gesetz, und indem die Parabel dies tut, ist sie selbst ein Türhüter vor dem Gesetz.

Wenn die Erzählung erfolgt, ist sie für Josef $\mathrm{K}$. allerdings nicht ganz neu, einerseits, weil seine eigene Lage jener des Mannes vom Lande in Vielem gleicht, andererseits aber, weil der Erzählung eine weitere Türhüter-Konstellation unmittelbar vorangeht, diese als Parallel- oder Gegenszene vorbereitend. Bei seinem Rundgang durch den dunklen Dom stösst K., mit einer Taschenlampe ausgerüstet, in einer Seitenkapelle auf ein Altarbild, vor dem »störend « das ewige Licht »schwebt[...]« (281):

"Das erste was K. sah und zum Teil erriet, war ein grosser gepanzerter Ritter, der am äussersten Rande des Bildes dargestellt war. Er stützte sich auf sein Schwert, das er in den kahlen Boden vor sich - nur einige Grashalme kamen hie und da hervor - gestossen hatte. Er schien aufmerksam einen Vorgang zu beobachten, der sich vor ihm abspielte. Es war erstaunlich, dass er so stehen blieb und sich nicht näherte. Vielleicht war er dazu bestimmt, Wache zu stehen. K., der schon lange keine Bilder gesehen hatte, betrachtete den Ritter längere Zeit, trotzdem er immerfort mit den Augen zwinkern musste, da er das grüne Licht der Lampe nicht vertrug. Als er dann das Licht über den übrigen Teil des Bildes streichen liess, fand er eine Grablegung Christi in gewöhnlicher Auffassung, es war übrigens ein neueres Bild. Er steckte die Lampe ein und kehrte wieder zu seinem Platz zurück.» (281)

Die Nähe der beiden Szenen ist offensichtlich. K.s Entdeckung stellt im Medium des Bildes eine christliche Variante zur jüdisch akzentuierten Parabel dar. An die Stelle des Gesetzes tritt die Grablegung Christi »in gewöhnlicher Auffassung", der Türhüter wird (Mt 27,63ff. folgend) ein Ritter, der Mann vom Lande hat, wenig erstaunlich, seine Entsprechung in Josef K., der die Szene betrachtet. 
Die religions- und medienbezogenen Differenzen werden allerdings von weiteren subtilen Verschiebungen begleitet. Anders als der Türhüter dem Gesetz, ist der Ritter dem »Vorgang« der Grablegung zugewandt. Anders als der Türhüter den Glanz wird er den zu verhindernden Versuch eines Diebstahls des Korpus - bzw. die Auferstehung - sehen. Und anders als der Mann vom Lande ist der Betrachter K. nicht »im Bild«. Der Parabel wiederum entsprechend gilt aber auch K.s Interesse nicht dem Gehüteten, sondern dem Hüter, den er »ängere Zeit« studiert, das Licht hingegen nur beiläufig »über den übrigen Teil des Bildes streichen « lässt, um sich dann gleich auf seine technische Machart zu konzentieren. Und wie der Mann vom Lande hat auch K. Probleme mit den Augen.

Dass sich K. zunächst intuitiv zur Figur des Mannes vom Lande hingezogen fühlt und in der Auslegung für ihn Stellung bezieht, ist verständlich. Darüber hinaus bildet das Gespräch, in dessen Verlauf die Parabel erzählt wird, deren Konstellation jedoch ein weiteres Mal ab: Vor dem Gesetz ereignet sich »im Dom«. Wie dem Mann vom Lande, so wird auch K. von oben ein Platz angewiesen; "mit dem scharf gesenkten Zeigefinger" weist der Geistliche "auf eine Stelle knapp vor der Kanzel« (287). In der Position des Gesetzes erscheint diesmal - die Parabel. Josef K. will verstehend in sie eindringen, wird vom Türhüter-Geistlichen jedoch unter Verweis auf die Vielzahl der möglichen "Erklärung[en]« (301) abgewiesen. Die Beugebewegung des Türhüters kehrt hier wieder in Gestalt des Abstiegs des Geistlichen von der Kanzel; die scheinbar abschlägige Antwort auf die letzte Frage des Mannes vom Lande (gerichtet auf den Widerspruch zwischen der Allgemeinheit des Gesetzes und dem Ausbleiben anderer Eintrittsuchender) entspricht der ausbleibenden bzw. in Gestalt der vieldeutigen Parabel vorgebrachten Antwort auf K.s Hoffnung, vom Geistlichen zu erfahren, "wie man aus dem Process ausbrechen, [...] wie man ausserhalb des Processes leben könnte" (291; meine Hervorhebungen, A. M.). K.s anfängliches Beharren darauf, im Grunde ja nichts anderes als ein kulturinteressierter Fremder zu sein, entfällt. Wie der Mann vom Lande ist auch er ein Bittsteller.

\section{Seitwärts vor dem Gesetz: der Kommentar}

Deutlich wird: Die Parabel steht nicht isoliert im Gefüge des Romans, vielmehr sind ihr verschiedenste Parallelszenen an die Seite gestellt, sei es in der globalen Ähnlichkeit der Schicksals K.s mit dem 
des Mannes vom Lande, sei es in der Anlage des Gesprächs K.s mit dem Geistlichen, sei es im Medium der Bildbetrachtung K.s oder sei es - dies blieb hier unerwähnt - in den Versionen der TürhüterSzene, die an verschiedenen Stellen in den Roman eingelassen sind (etwa im Leid des »kleinen Advokaten«; vgl. 158f). Sie alle scheinen sich wechselseitig zu kommentieren, wobei die dreistellige Struktur Mann vom Lande - Türhüter - Gesetz immer wieder neue Besetzungen erfährt und im Zuge einer Umbesetzung auch die Stellen selbst modifiziert werden. Die Raumsemantik im ersten und im zweiten Sinn verschlingen sich ineinander: Der Parabeltext kann sowohl die Position des Gesetzes einnehmen als auch die des Türhüters vor dem Gesetz. Die wiederholten in sich dynamischen Refigurationen erlauben es nicht, eine Fassung auf eine andere $\mathrm{zu}$ reduzieren. Unverkennbar bleibt jedoch, dass der Parabel ein privilegierter Status zukommt; sie scheint eine Art Urszene zu sein, alle anderen ihre Versionen, die sich als Kommentarschichten an ihre Seite stellen. Das ist doppelt zu begründen, textextern unter Rekurs auf Kafkas eigene Wahrnehmung der »Legende«, aber auch textimmanent. War Kafkas Verhältnis zu seiner eigenen literarischen Produktion bekanntlich ein äusserst gespaltenes, so entfällt diese Ambivalenz im Fall der Parabel für einmal fast vollständig. Der Tagebucheintrag vom 13. Dezember 1914 spricht von seinem "Zufriedenheits- und Glücksgefühl«, das er »besonders der Legende gegenüber « ${ }^{11}$ empfinde. Offenbar schien ihm das Anliegen seines Schreibens - an anderer Stelle als "Ansturm gegen die Grenze « ${ }^{12}$ bezeichnet - hier in gültiger Weise gestaltet. Innerhalb des Romans sticht die Parabel schliesslich nicht nur deshalb hervor, weil sie den gesamten Roman im Sinne einer mise en abyme abbildet, sondern darüber hinaus Gegenstand ausführlicher Erörterung wird. Stellen die erwähnten Szenen implizite Kommentare dar, so werden sie hier explizit. Die Offenheit der szenischen Kommentare - Wie wäre die Bildbetrachtung K.s im Detail auf die Parabel zu beziehen? - wird hier zum Programm, die Pluralität der Lesarten wird geradezu zelebriert. Der talmudischen Kommentartradition ähnlich reiht der Geistliche eine »Meinung" gleich gültig an die andere: "Die Erklärer sagen hiezu« (297), »[m]anche gehn sogar in dieser Art der Erklärung noch weiter und meinen« (298), »[a]ndere sagen zwar« (299), »[d]arüber gehn die

\footnotetext{
${ }^{11}$ Kafka, Tagebücher 1910-1923, 326.

12 Man vergleiche den nicht nur in dieser Hinsicht auf die Parabel beziehbaren Tagebucheintrag vom 16. Januar 1922, ebd. 405f.
} 
Meinungen auseinander« (301), »[d] arin aber sind viele einig« (301) etc. K.s anfängliche Sicherheit des Urteils - »Der Türhüter hat also den Mann getäuscht [...].«(295) - gerät durch der Fülle der Auslegungsmöglichkeiten ins Wanken; die »einfache Geschichte« wird »unförmlich« (303).

Die vorgetragenen »Meinungen« brauchen hier nicht im Einzelnen referiert $\mathrm{zu}$ werden, es genügt, die beiden hermeneutischen Maximen der »Erklärer« zu erinnern: »Richtiges Auffassen einer Sache und Missverstehn der gleichen Sache schliessen einander nicht vollständig aus. [...] Du musst nicht zuviel auf Meinungen achten. Die Schrift ist unveränderlich und die Meinungen sind oft nur ein Ausdruck der Verzweiflung darüber.«(297f) Die Gleichzeitigkeit von Verstehen und Missverstehen hält die Kommentierung in Gang, sie ist unabschliessbar, da die Widersprüchlichkeit der Meinungen immer neue Meinungen hervorbringt. Wenn sie abbricht, dann also nicht, weil sie die unveränderliche »Schrift« erschöpft hätte und ein »Endurteil« (303) erreicht wäre, sondern weil der Kommentierende, wie K., im Moment »zu müde« ist, »um alle Folgerungen der Geschichte übersehn zu können« (303).

Der Kommentar weiss, dass er nie in die Schrift eindringen und diese ablösen wird. Daher positioniert er sich nicht frontal, sondern bettlerhaft seitwärts vor ihr. Vielleicht lässt sich die Episode in Vor dem Gesetz (die ja ihrerseits vor dem Gesetz steht), als - zwiespältige - Allegorie des Kommentars lesen. Zwiespältig, weil der Kommentar offensichtlich Gefahr läuft, das Kommentierende aus den Augen zu verlieren, zwiespältig aber vor allem, weil offen bleibt, ob da überhaupt ein zu Kommentierendes ist. Der Lehre der Parabel, die Bewertung ihres Personals, hängt letztlich einzig und allein an der Bewertung des Glanzes, der aus dem Gesetz bricht. Wenn man ihn nicht für einen Schein hält, bedeutet dies: Man muss nicht »den Mechanismus kennen«, man muss sich vor dem Gesetz hinsetzen, seitwärts.

_ Andreas Mauz ist Assistent am Institut für Hermeneutik und Religionsphilosophie. 